\title{
Design and fabrication of a large vertical-travel silicon inchworm microactuator for the Advanced Segmented Silicon Space Telescope
}

Eui-Hyeok Yang, Richard G. Dekany, Stephen Padin

Eui-Hyeok Yang, Richard G. Dekany, Stephen Padin, "Design and fabrication of a large vertical-travel silicon inchworm microactuator for the Advanced Segmented Silicon Space Telescope," Proc. SPIE 4981, MEMS Components and Applications for Industry, Automobiles, Aerospace, and Communication II, (16 January 2003); doi: 10.1117/12.476282

EDIE Event: Micromachining and Microfabrication, 2003, San Jose, CA, United States 


\title{
Design and Fabrication of a Large Vertical Travel Silicon Inchworm Microactuator for the Advanced Segmented Silicon Space Telescope
}

\author{
Eui-Hyeok (EH) Yang $*^{\mathrm{a}}$, Richard Dekany $^{\mathrm{b}}$ and Steve Padin ${ }^{\mathrm{b}}$ \\ ${ }^{a}$ Jet Propulsion Laboratory, California Institute of Technology \\ 4800 Oak Grove Drive, Pasadena, CA 91109 \\ *Eui-Hyeok.Yang@jpl.nasa.gov ph: +1-818-354-7059 fx: +1-818-393-6047 \\ ${ }^{\mathrm{b}}$ California Institute of Technology, Division of Physics, Mathematics, and Astronomy \\ M/S 105-24, Pasadena, CA 91125
}

\begin{abstract}
Future concepts for ultra-large lightweight space telescopes include the telescopes with segmented silicon mirrors. This paper describes a proof-of-concept inchworm actuator designed to provide nanometer resolution, high stiffness, large output force, long travel range, and compactness for ultraprecision positioning applications in space. A vertically actuating inchworm microactuator is proposed to achieve large actuation travel by incorporating compliant beam structures within a silicon wafer. An inchworm actuator unit consists of a piezoelectric stack actuator, a driver, a pair of holders, a slider, and a pair of polymer beams connected to a centrally clamped flexure beam. Deep reactive ion etch experiments have been performed for constructing the actuator.
\end{abstract}

Keywords: Advanced Segmented Silicon Space Telescope (ASSiST), Piezoelectric Actuator, Inchworm Actuator, Bistable Flexure Beam, Large Vertical Travel

\section{INTRODUCTION}

Advanced Segmented Silicon Space Telescope (ASSiST) is a novel space telescope concept that derives high functionality from passive spacecraft components [1]. We propose to achieve considerable reductions in primary mirror mass through the use of thin ( 400 micron) silicon wafers, eliminating the need for a heavy, back plane support structure. Rather, we exploit the micromachining capabilities of silicon processing technology to achieve sophisticated control of a highly segmented mirror using high-bandwidth, high-stroke actuators. Ultimately, these actuators can be built directly into the mirror segment, resulting in an integrated optics package. Thus, a single segment can perform both the traditional light focusing function of a telescope as well as the control functions, and quite possibly the space deployment functions. Although our long-term vision of silicon-based space telescopes includes even greater functionality, such as solar-based power generation, for now we plan to concentrate on the development of robust actuators as the initial first step to proving the ASSiST concept. The basic configuration for ASSiST is presented in Figure 1. The development of MEMS (Micro Electro Mechanical Systems) actuators and sensors enables this concept (large segmented telescope mirrors of unprecedented area density). Substantial mass reduction is achieved by integrating most of the functionality of these large structures into the individual optical segments. The conceptual use of MEMS actuators in the phasing of a segmented optical system is shown in Figure 2. We propose a MEMS-based actuation system, in which a set of MEMS actuators, such as miniaturized inchworm actuators, controls the optical surface figure of the primary optics. A MEMS-based electrostatic linear inchworm motor has been previously reported [2]. These actuators move horizontally on the surface of a silicon wafer, and thus cannot provide vertical motion. A mesoscale inchworm actuator has also been developed [3]. However, actuators suitable for the ASSiST application require further miniaturization. A thermal inchworm actuation concept has also been presented in literature, with $50 \mu \mathrm{N}$ actuation force and $0.2 \mu \mathrm{m}$ travel resolution [4]. However, significantly more precision, as well as rapid actuation with a larger force is required. Although a proof-of-concept MEMS-based vertical inchworm 
actuator has been reported [5], to our knowledge, none of the above approaches provide the large vertical travel actuation ( hundreds of microns) that is required. We propose here a novel concept for a MEMS-based piezoelectric inchworm microactuator capable of large vertical travel ( hundreds microns) with precision step motion. Our inchworm actuator technology is achieved by combining piezoelectric-driving and electrostatic-clamping actuation within silicon microstructures.

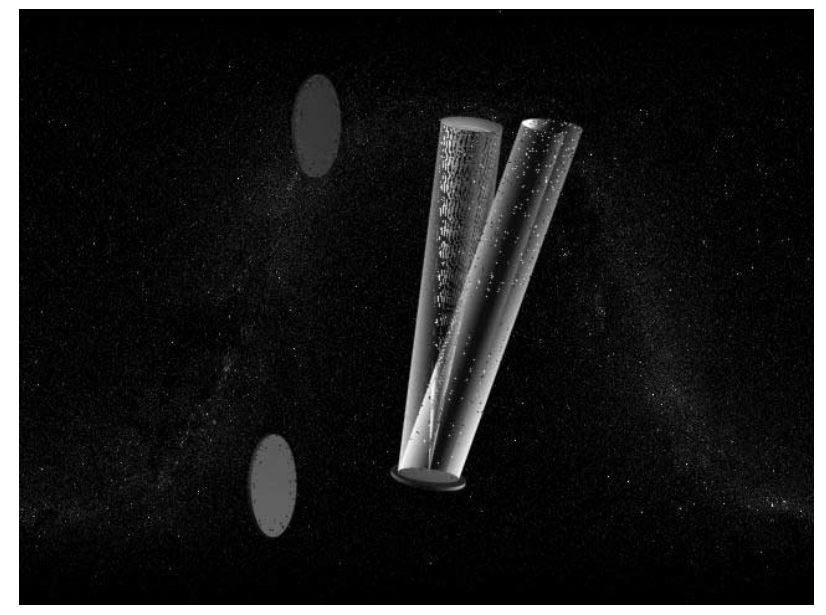

Figure 1: Spacecraft formation flying geometry for ASSiST. Starlight entering from the top-right is collected by a Schmidt corrector mirror (bottom) then focused by the F/10 primary mirror (top). Also shown are two large sunshades (at left), which may be inflatable. The instrument bus resides behind the corrector mirror.

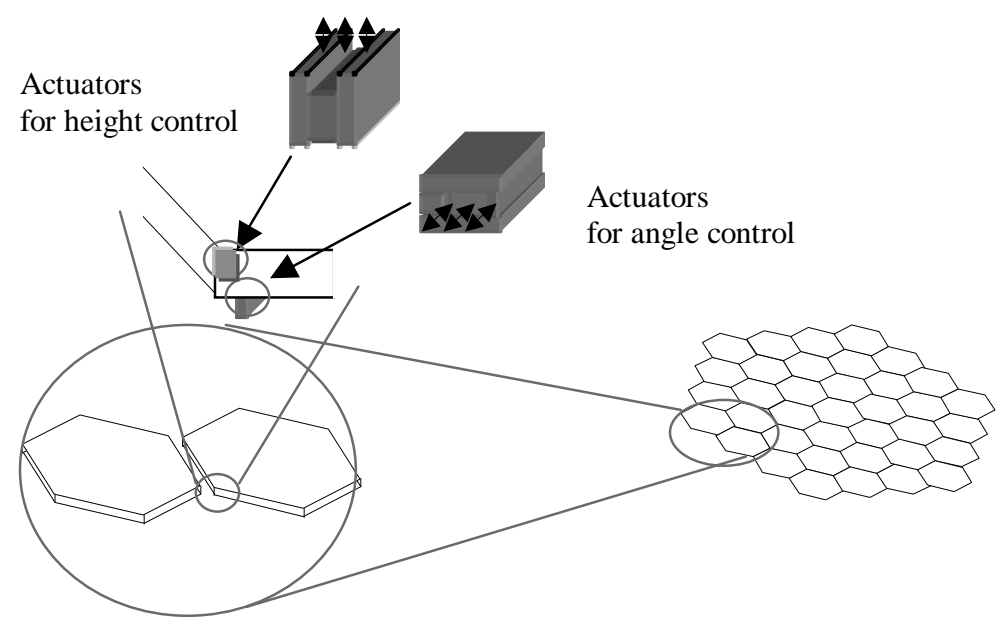

Figure 2: The role of MEMS technology in segmented mirror actuation is depicted. In this example two actuators are shown, one controlling relative segment height (or piston) and the other controlling the relative dihedral angle between segments.

\section{PRIMARY MIRROR AND ACTUATION}

\subsection{Primary Mirror Configuration}

The current design for the ASSiST primary mirror consists of a sheet composed of $30 \mathrm{~cm}$ diameter, $400 \mu \mathrm{m}$ thick, hexagonal silicon wafers. The mirror is made into hexagonal rafts of approximately 100 segments, each mounted on a deep frame to provide stiffness on $3 \mathrm{~m}$ scales. Conventional actuators (e.g. motor-driven screws) adjust inter-raft 
piston and tilt about the raft edges to control spatial scales larger than a raft. This is equivalent to the control of a ground-based segmented mirror telescope, but the raft frames are much simpler and lighter than a ground-based mirror cell. Autonomous control of each raft is an obvious hierarchy that simplifies the overall control of the mirror. The segments within a raft are mounted on $10 \mathrm{~mm}$ deep silicon frames, which would limit the segment warp and provide mounting surfaces for the actuators. The frames also distribute actuator forces to reduce local deformation (actuator "print-through"). Control forces would be applied between adjacent segments, with a control range of a few times 10 $\mu \mathrm{m}$ to accommodate misalignments during segment assembly. The forces required to correct warps of $10 \mu \mathrm{m}$ over a segment are on the order of $1 \mathrm{mN}$, which is well within the capabilities of the actuators described in this paper. The yield strength of the actuators allows acceleration of the telescope up to $10^{-7} \mathrm{rad} \mathrm{s}^{-2}$ (cf. $10^{-4} \mathrm{rad} \mathrm{s}^{-2}$ for a continuous 3$\mathrm{m}$ diameter, $200 \mu \mathrm{m}$ thick silicon sheet), making pointing changes of order $1 \mathrm{rad} \mathrm{hr}^{-1}$ possible.

A key element of the success of this concept relies heavily on the ability to develop very precise, very stiff, high bandwidth actuators using MEMS processing techniques. While these actuators may be used in the classical sense to push against some form of back plane, MEMS technology makes possible an interesting option of phasing a mirror without the need for a heavy backing structure. Rather, segment tip/tilt would be maintained through forces conducted within the plane of individual segments, while segment piston, which is purely a relative motion, would be maintained through forces normal to the segments. Thus the figure control actuators provide a unique adaptive structural linkage between the many individual segments. Furthermore, an important capability is added because of the small-scale sizes of MEMS actuators; typically tens of microns, they are capable of operation at bandwidths of tens of kilohertz thus providing significant mechanical stiffness for the large segmented ASSiST mirrors. Although these same scales size limit the forces that MEMS actuators can exert, this limitation is not severe when positioning very thin (and thus low moment of inertia) segments in Space.

\subsection{Mechanical Issue}

The ASSiST primary has no mirror cell to provide reaction forces for the segment actuators. Although this scheme reduces the mass of the primary, position control forces must be applied between segments. Each segment edge requires piston and tilt actuators to uniquely determine the shape of the mirror. The construction of piston actuators alone is relatively straightforward, however the addition of tilt actuators requires a combination of hinges and linear actuators (see Fig. 3). The hinges have to be fairly stiff in piston mode, and so serial operation of hinge and piston actuators is preferred. Such operation is achieved by mounting the piston actuators on beam flexures, which provide the hinge function. The tilt actuators are also

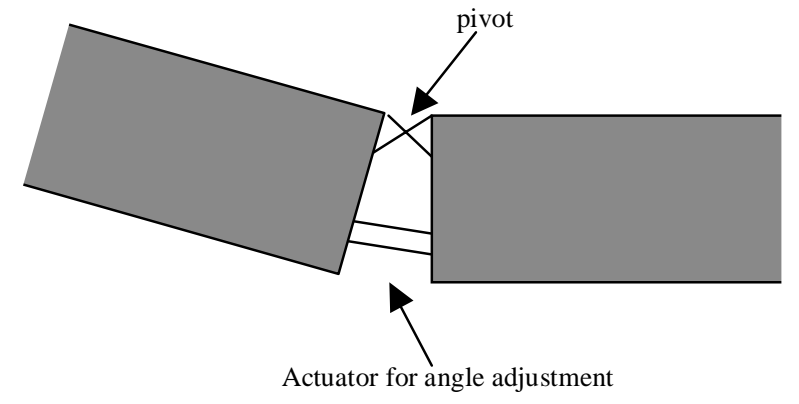

Figure 3: Segment configuration for the edge actuation concept. mounted on flexures to accommodate piston and tilt motions. Flexures are integrated with the MEMS actuators, or included as separate sub-assemblies, which might be more appropriate for a prototype mirror.

\section{INCHWORM STRUCTURES FOR LARGE TRAVEL ACTUATION}

\subsection{Design}

The inchworm actuator motion is facilitated via sequential actuation of actuation elements. The inchworm actuator is essentially a linear motor with extremely fine resolution. The design proposed in this paper consists of a piezoelectric stack actuator, a silicon driver, a pair of holders, a slider, and a pair of polymer beams connected with a centrally-clamped bi-stable flexure beam as shown in Figure 4. All the parts except for the piezoelectric stack and the polymer beams are made from a $350 \mu \mathrm{m}$ thick silicon-on-insulator (SOI) wafer. The estimated clamping force is $1 \mathrm{~N}$. 
Since the motion step size (step resolution) is critically dependent on the smoothness and straightness of the sidewalls, a robust fabrication process for smooth, vertical sidewalls needs to be developed.

The ultimate device is expected to have the following specifications:

- $\quad$ Operation up to $1 \mathrm{kHz}$

- Operation at temperatures at or below $77 \mathrm{~K}$ with $15-20 \%$ room temperature efficiency

- $\quad 250 \mu \mathrm{m}$ total travel with nanometer-level precision

- Electrostatic holding force between segments of approximately 1 N (@ $100 \mathrm{~V})$

- Mean steady-state power consumption at $100 \mathrm{~mW}$ per actuator

Our initial efforts have focused on performing Deep Reactive Ion Etching (DRIE) experiments for the microfabrication of the MEMS inchworm actuators. Several technical issues were encountered during the course of these experiments. (See the SEM images shown in Figure 5.) Additional process development is required in order to address these issues. In particular, a robust DRIE process needs to be developed for obtaining smooth, vertical sidewalls required for nanometer step resolution for the inchworm actuation.
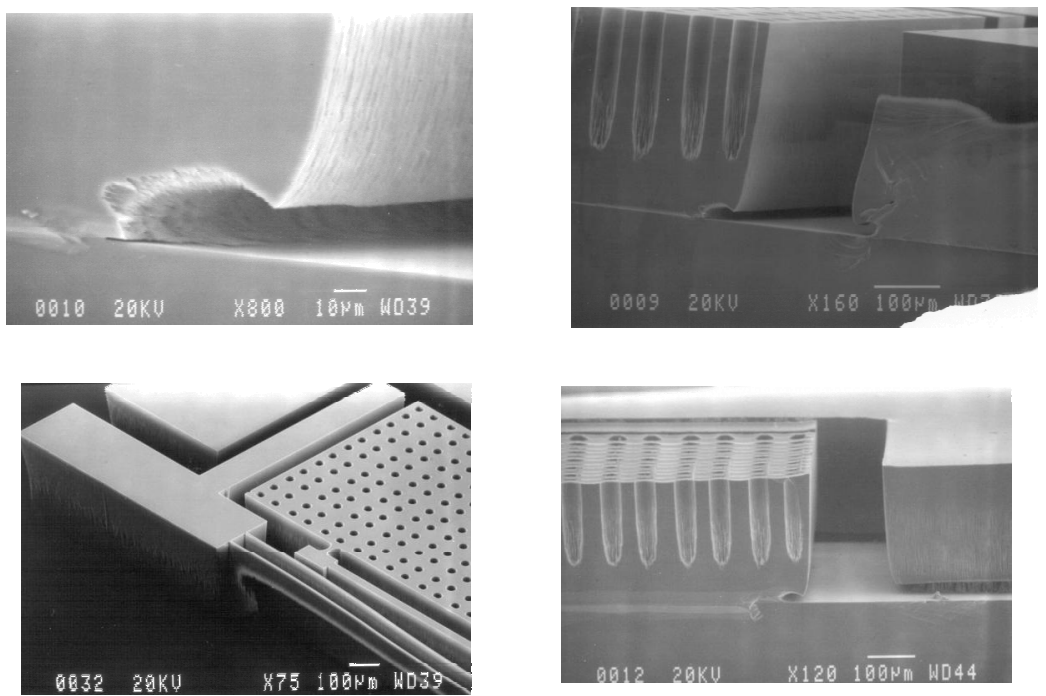

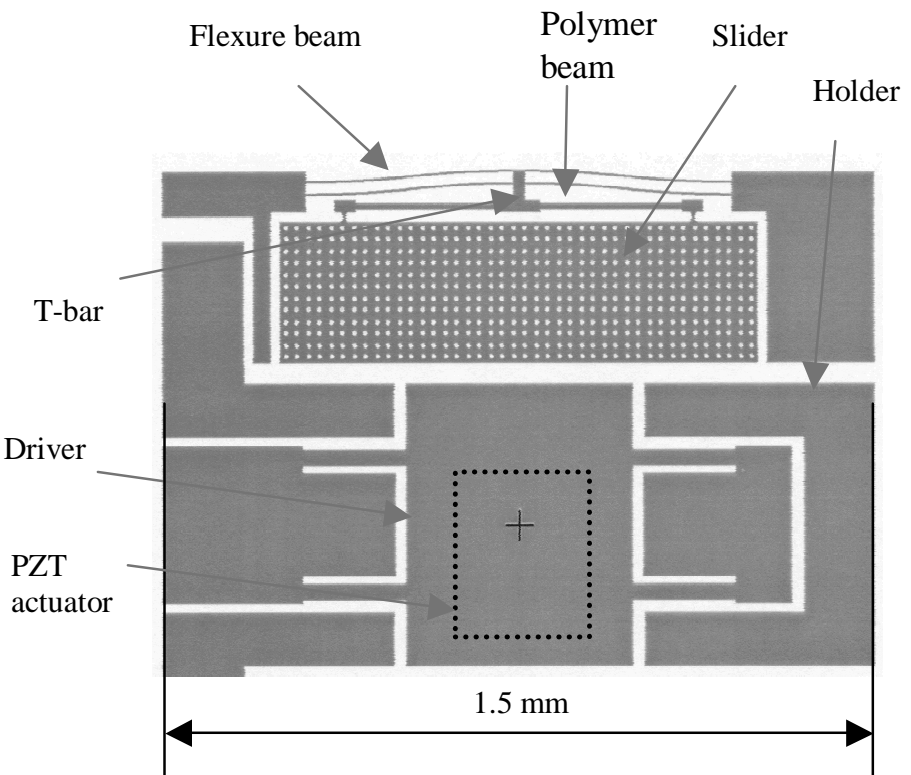

Figure 4: Top view of the actuator

Figure 5: SEM micrographs of the microfabricated actuator structures. The DRIE challenges, such as "footing", over-etching and non-uniform etching, remain to be overcome.

\subsection{Bi-stable Beam for Optimized Electrode Gap}


The minimum width of trenches etched in silicon via the DRIE process is determined primarily by the achievable aspect ratio of the etching process, which is typically $25: 1$. Thus, trench widths on the order of 15 25 microns, are obtained for silicon microstructures fabricated from 350 500-micron-thick wafers. The use of such wide trenches for electrostatic clamping could result in defective actuation with attendant high voltage operation. Therefore, a centrally clamped, bi-stable flexure beam is incorporated in the design in order to reduce the gap between the slider and the driver/holder. The flexure beam connected to the slider is designed to snap towards the driver, via a mechanical buckling process, and thereby reduce the initial gap (for the electrostatic actuation) between the slider and driver/holder. A considerable amount of work remains to be done in order to optimize the design and fabrication process for this type of flexure beam structure. Estimates of total travel greater than $250 \mu \mathrm{m}$ have been obtained for polymeric materials such as Teflon, Parylene and Silicone rubber (low Young's moduli) when used for the compliant beam structures.

\subsection{Actuation Mechanism}

The inchworm actuation mechanism works as follows (See Figure 6.):

1) The centrally clamped flexure beam is made to snap down to the $2^{\text {nd }}$ stable position by means of an external probe. The snap down process makes the T-bar push the slider toward the driver. The polymeric beam is compliant enough to accommodate the shear strain required for this action. The snap down insures that the gap between the slider and the driver is within a couple of microns.

2) The slider is then electrostatically clamped to the driver. The driver is subsequently pushed upward by the piezoelectric stack actuator directly bonded to the driver. The slider also moves upward with the driver.

3) The slider is then electrostatically clamped by the fixed holders. Next, the slider is released from the driver. The released driver moves downward via piezoelectric actuation, while the slider remains clamped to the fixed holders.

4) Vertical inchworm actuation is achieved by repeating these steps (step 2-3).

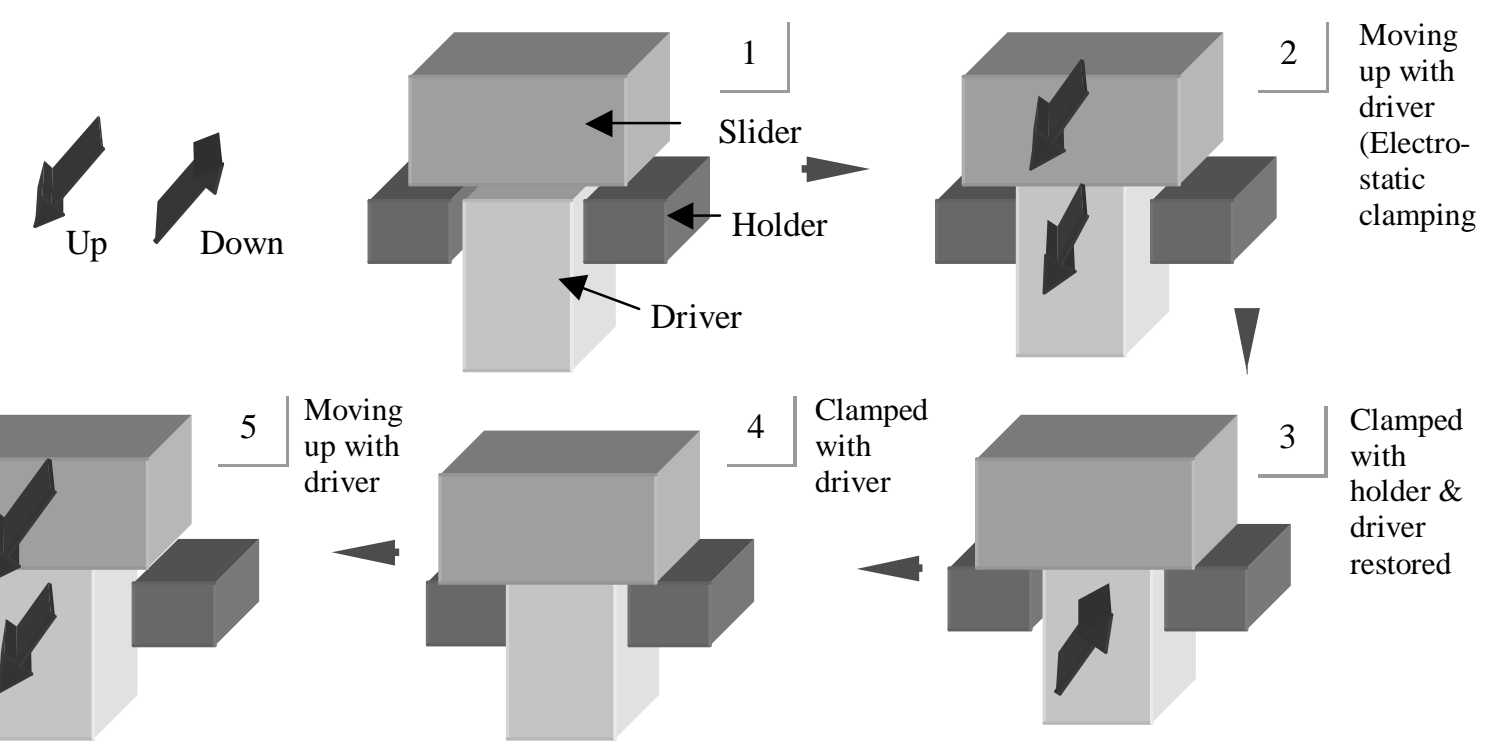

Figure 6: Actuation sequence of the inchworm actuation. 1-2) The slider is electrostatically clamped to the driver. The driver is moved upward (out of the page) by the piezoelectric stack actuator bonded to the bottom of the driver. 3) The slider is then clamped with the holders. The slider is subsequently released from the driver. The separated driver moves downward (into the page) via piezoelectric actuation, while the slider is clamped to the fixed holders. 4) The slider is clamped once again to the driver while the slider is still clamped to the holders. Next, the slider is released from the holders. 5) The slider is then moved upward by the driver. Inchworm actuation is achieved by repeating these steps (step 2-5). 


\section{CONCLUSIONS}

One of the key drivers for segment and actuator concepts for ASSiST is the segment configuration for the primary and corrector mirrors. Depending on segment geometry, assuming $300 \mathrm{~mm}$ diameter wafer technology, ASSiST the total number of segments required are between 10,000 and 15,000. Thus, MEMS actuation technology is absolutely essential in order to realize large structures such as the $30 \mathrm{~m}$ diameter primary mirror. A MEMS-based vertical inchworm microactuator has been proposed to achieve the large travel actuation required for this concept. Compliant polymer beams and bi-stable flexure beams have been designed to achieve the inchworm structure with large vertical travel actuation. The inchworm actuator motion can be created through sequential activation of actuation elements. The MEMS inchworm actuator discussed in this paper will be capable of a very large stroke of about 250 microns, with a resolution of a few nanometers, and will occupy only about $2 \mathrm{~mm}^{3}$, and weigh only about 10 milligrams. The ultra lightweight large stroke actuator technology proposed in this paper opens up a whole new paradigm in microactuation technology, and will enable the ultra lightweight actuated mirrors for future generations of space telescopes.

\section{ACKNOWLEDGEMENTS}

The Jet Propulsion Laboratory, California Institute of Technology carried out the research described in this paper under a contract with the National Aeronautics and Space Administration.

\section{REFERENCES}

1. R. Dekany, et al., "Advanced Segmented Silicon Space Telescopes (ASSiST)," SPIE Int'l symp. On Astronomical Telescopes and Instrumentation, Adaptive Optical System Technologies II, 22-28 August 2002, Waikoloa, Hawaii, USA.

2. R. Yeh, et al., "Single Mask, Large Force, and Large Displacement Electrostatic Linear Inchworm Motors," IEEE Micro Electro Mechanical Systems (MEMS) 2001 Conference, Interlaken, Switzerland, Jan. 21-25, 2001, p. 260.

3. Q. Chen, et al., " Mesoscale Actuator Device with Micro Interlocking Mechanism," IEEE Micro Electro Mechanical Systems (MEMS) 1998 Conference, Heidelberg, Germany, Jan. 1998, p. 384.

4. H.N. Kwon et al., "Characterization of a Micromachined Inchworm Motor with Thermoelastic Linkage Actuators," IEEE Micro Electro Mechanical Systems (MEMS) 2002 Conference, Las Vegas, Jan. 2002, p. 586.

5. S. Konishi, et al., "Vertical Motion Microactuator based on the Concept of ECLIA," IEEE Micro Electro Mechanical Systems (MEMS) 2002 Conference, Las Vegas, January 2002, p. 602.

6. J. Qiu, et al., "A Centrally-Clamped Parallel-Beam Bistable MEMS Mechanism," IEEE Micro Electro Mechanical Systems (MEMS) 2001 Conference, Switzerland, Jan. 2001, p. 353. 